\title{
The efficacy of microsurgery in the treatment of cerebral aneurysm rupture and its effect on NF-кB, MCP-1 and MMP-9
}

\author{
XINTONG ZHANG ${ }^{1,2}$, LEI CHEN ${ }^{3}$, FENG ZHENG ${ }^{4,5}$ and YANLI DU ${ }^{1}$ \\ ${ }^{1}$ The Second Affiliated Hospital of Soochow University, Suzhou, Jiangsu 215004; \\ ${ }^{2}$ The Second Clinical Medical School of Inner Mongolia University for the Nationalities, Yakeshi, \\ Inner Mongolia 022150; ${ }^{3}$ Hulunbuir People's Hospital, Hulunbuir, Inner Mongolia 021000, P.R. China; \\ ${ }^{4}$ Department of Neurosurgery, University Hospital of Cologne, D-50937 Cologne, Germany; ${ }^{5}$ Department of \\ Cerebrovascular Disease, Affilliated Hospital of Zunyi Medical College, Zunyi, Guizhou 563000, P.R. China
}

Received March 31, 2017; Accepted August 3, 2017

DOI: 10.3892/etm.2017.4928

\begin{abstract}
The clinical efficacy of microsurgical neck clipping for the treatment of cerebral aneurysm rupture and its effect on serum nuclear factor $\kappa$-light-chain-enhancer of activated $\beta$ cells (NF- $\mathrm{kB}$ ), monocyte chemoattractant protein-1 (MCP-1) and matrix metalloproteinase-9 (MMP-9) levels were investigated. A total of 56 patients with first occurrence of cerebral aneurysm rupture were enrolled from June 2015 to June 2016. These patients were divided into control (25 patients) and observation groups (31 patients) according to treatment received. The patients in the control group were treated with interventional embolization and extraventricular drainage, while the patients in the observation group were treated with microsurgical neck clipping. Serum NF- $\mathrm{B}, \mathrm{MCP}-1$ and MMP-9 levels were measured by ELISA prior to the operation and at $6 \mathrm{~h}$ post-operation. Clinical effects were compared at the 6 -month follow-up. There was no significant difference in the success rate of the operation between the two groups $(p>0.05)$. The incidence of complications in the observation group was significantly lower than that in the control group $(\mathrm{p}<0.05)$. The Glasgow Outcome Scale score was significantly improved in the observation group $(\mathrm{p}<0.05)$ compared with the control group. Serum NF-kB, MMP-9 and MCP-1 were significantly decreased in both groups at 6 and $24 \mathrm{~h}$ after operation, but the observational group showed significantly lower levels for all three proteins than the control group $(p<0.05)$. The application of early microsurgical neck clipping for the treatment of cerebral aneurysm rupture can reduce complications and improve clinical prognosis, and this may be related to a decrease in
\end{abstract}

Correspondence to: Dr Yanli Du, The Second Affiliated Hospital of Soochow University, 1055 Sanxiang Road, Suzhou, Jiangsu 215004, P.R. China

E-mail:duv3bj@163.com

Key words: microsurgery, interventional embolization, cerebral aneurysm, NF-кB, MMP-9, monocyte chemoattractant protein-1 serum inflammatory response-related factors $(\mathrm{NF}-\kappa \mathrm{B}$ and MCP-1) and MMP-9.

\section{Introduction}

Cerebral aneurysm is the main cause of spontaneous subarachnoid hemorrhage (SAH), with an incidence of rupture of approximately $1-5 \%$, which is only lower than the incidence of hypertensive intracerebral hemorrhage and cerebral thrombosis. The 30-day mortality rate is as high as 45 and $30 \%$ of survivors have moderate to severe neurological dysfunction (1). Studies have shown that chronic inflammation and pathological remodeling of the extracellular matrix may be an important cause of aneurysm (2). The elastic protease infusion-induced experimental animal aneurysm model is similar to human aneurysms at both morphological and pathological levels (3). Studies on abdominal aortic aneurysms have shown that the infiltration of various inflammatory cells can especially promote the secretion of matrix metalloproteinases (MMPs).

MMP-9, which in turn accelerates the degradation of elastic and collagen fibers in the arterial wall (4). Nuclear factor $-\kappa \mathrm{B}(\mathrm{NF}-\kappa \mathrm{B})$ can regulate the expression of a variety of genes involved in the inflammatory response (5). Monocyte chemoattractant protein-1 (MCP-1) is a member of the chemokine CC subfamily and one of the monocyte-macrophage inducing chemokines. As a downstream target protein of $\mathrm{NF}-\kappa \mathrm{B}, \mathrm{MCP}-1$ plays a role in inducing the inflammatory cascade.

There is no common understanding on the surgical timing and surgical methods used for the treatment of acute cerebral aneurysm rupture. Traditional treatment methods include decompressive craniotomy, paracentesis and interventional embolization. Early microsurgical neck clipping can significantly reduce the incidence of rupture and prevent postoperative cerebral vasospasm (6). Early microsurgical neck clipping has a positive effect in cases of high-risk aneurysm rupture (7).

The present study further evaluated whether the effect of microsurgery in the treatment of cerebral aneurysm rupture was associated with changes in the serum levels of $N F-\kappa B$, MCP-1 and MMP-9. 
Table I. Comparison of baseline characteristics.

\begin{tabular}{lcccr}
\hline Characteristics & $\begin{array}{c}\text { Control group } \\
(\mathrm{n}=25)\end{array}$ & $\begin{array}{c}\text { Observation group } \\
(\mathrm{n}=31)\end{array}$ & $\mathrm{t} / \mathrm{\chi}^{2}$ & P-value \\
\hline Sex (male/female) & $13 / 12$ & $16 / 15$ & 0.001 & 0.977 \\
Age (years) & $52.6 \pm 9.5$ & $53.3 \pm 8.7$ & 0.096 & 0.924 \\
Onset time (h) & $10.2 \pm 3.5$ & $12.1 \pm 3.6$ & 0.325 & 0.768 \\
Hypertension (cases, \%) & $6(24.0)$ & $8(25.8)$ & 0.024 & 0.877 \\
Diabetes (cases, \%) & $4(16.0)$ & $6(19.4)$ & 0.000 & 1.000 \\
Hunt-Hess grading (cases, \%) & & & 0.119 & 0.730 \\
Level I-II & $7(28.0)$ & $10(32.3)$ & & \\
Level III-V & $18(72.0)$ & $21(67.7)$ & & \\
Bleeding site (cases, \%) & & & & \\
Anterior communicating artery & $12(48.0)$ & $15(48.4)$ & & \\
Posterior communicating artery & $6(24.0)$ & $7(22.6)$ & & \\
$\quad$ Middle cerebral artery & $7(28.0)$ & $9(29.0)$ & & \\
Aneurysm diameter (mm) & $8.8 \pm 1.6$ & $8.7 \pm 1.5$ & 0.152 & 0.991 \\
Intracerebral hematoma (cases, \%) & $8(32.0)$ & $13(41.9)$ & 0.583 & 0.445 \\
Cerebroventricular haemorrhage (cases, \%) & $6(24.0)$ & $8(25.8)$ & 0.024 & 0.877 \\
\hline
\end{tabular}

\section{Patients and methods}

Patient information. A total of 56 patients with first occurrence of cerebral aneurysm rupture were continuously selected from June 2015 to June 2016. Emergency surgery indications were detected in the patients by CTA examination. This study was approved by the Ethics Committee of the Second Affiliated Hospital of Soochow University. Signed written informed consents were obtained from the patients and/or guardians. Exclusion criteria were: Presence of combined brain tumor, cerebral vascular malformations, arteriovenous fistula and hypertensive intracerebral hemorrhage; presence of serious disturbance of consciousness, intracranial space-occupying lesion, serious condition and expected survival time $<24 \mathrm{~h}$; and presence of other underlying diseases which may affect the results.

The patients were divided into control (25 patients) and observation groups (31 patients) based on treatment received. The two groups were equivalent regarding key baseline characteristics (Table I).

Methods. In accordance with standard medical practices, the operations and procedures were performed by the same surgical and nursing team. Control group patients were treated with interventional embolization and extraventricular drainage. Intracranial pressure and blood pressure were controlled with gastrointestinal bleeding and cerebral vasospasm was prevented prior to surgery. General anesthesia was induced and the Seldinger technique was used to puncture the femoral artery. The bilateral internal carotid artery and vertebral artery were subjected to DSA examination. With the assistance of a microguide wire, the appropriate type of micro-coil was sent to complete the embolization. Embolization was confirmed by angiography. Patients with intracranial hematoma and ventricular hemorrhage were treated with extraventricular drainage.
The patients in the observation group were treated with microsurgical neck clipping. A Zeiss OPMI Pentero surgical microscope (E-Maxdent Co., Ltd., Beijing, China) and supporting equipment were used and the pterional approach was applied. The lateral scapula arachnoid and nearby blood vessels were carefully separated to fully expose the circle of Willis. The target vessel was separated and the neck of the tumor was completely clipped. No active bleeding was found after releasing the temporary blocking clip, indicating success of the procedure. To ensure tumor artery flow, a drainage tube was indwelled in the carotid cistern.

Observational index. The success and complication rates were compared between the two groups during a 6-month follow-up period. The Glasgow Outcome Scale (GOS) was used for scoring, and was defined as: Five points, patients recovered and returned to normal condition with mild defects; 4 points, with mild disability, patients could live independently and work under protection; 3 points, sober patients with severe disability, care is needed for daily life; 2 points, vegetative state with minimal response; and 1 point, death. The serum levels of NF- $\mathrm{B}, \mathrm{MMP}-9$ and MCP-1 were measured by ELISA prior to surgery and at 6 and $24 \mathrm{~h}$ after surgery. The reagents were obtained from Beyotime Institute of Biotechnology (Jiangsu, China). The microplate reader was purchased from Bio-Rad Laboratories, Inc. (Hercules, CA, USA). The operations were performed in strict accordance with the manufacturer's instructions. The experiments were performed three times and the average value was calculated.

Statistical analysis. Statistical analysis was performed using SPSS 20.0 statistical software (SPSS Inc., Chicago, IL, USA). Measurement data were expressed as mean \pm standard deviation (mean $\pm \mathrm{SD}$ ), and comparison between groups was made 
Table II. Surgery success rate and complication incidence (cases, \%).

Complication incidence

\begin{tabular}{lcccccc}
\cline { 3 - 6 } Groups & Success rate & Rebleeding & $\begin{array}{c}\text { Cerebrovascular } \\
\text { spasm }\end{array}$ & Infection & $\begin{array}{c}\text { Cerebrospinal } \\
\text { fluid leakage }\end{array}$ & Total \\
\hline Control $(\mathrm{n}=25)$ & $24(96.0)$ & $2(8.0)$ & $5(20.0)$ & $1(4.0)$ & $1(4.0)$ & $9(36.0)$ \\
Observation $(\mathrm{n}=31)$ & $28(90.3)$ & $1(3.2)$ & $2(6.5)$ & 0 & $1(3.2)$ & $4(12.9)$ \\
$\chi^{2}$ & 0.089 & & & & 4.142 \\
P-value & 0.766 & & & & 0.042 \\
\hline
\end{tabular}

Table III. GOS score (cases, \%).

\begin{tabular}{lccccc}
\hline Groups & 5 points & 4 points & 3 points & 2 points & 1 point \\
\hline $\begin{array}{l}\text { Control } \\
(\mathrm{n}=25)\end{array}$ & $8(32.0)$ & $11(44.0)$ & $4(16.0)$ & $1(4.0)$ & $1(4.0)$ \\
$\begin{array}{l}\text { Observation } \\
(\mathrm{n}=31)\end{array}$ & $19(61.3)$ & $8(25.8)$ & $2(6.5)$ & $1(3.2)$ & $1(3.2)$ \\
$\chi^{2}$ & & & & & \\
P-value & & & -2.073 & & \\
\hline
\end{tabular}

GOS, Glasgow Outcome Scale.

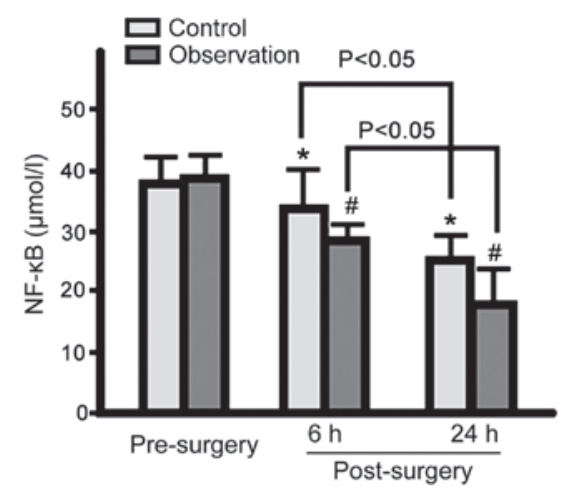

Figure 1. Serum NF- $\mathrm{kB}$ levels in the control and observation groups pre- and post-surgery. Serum NF- $\mathrm{B}$ levels in both groups gradually decreased at 6 and $24 \mathrm{~h}$ post-surgery, with serum NF-kB levels significantly lower in the observation group ( $\mathrm{p}<0.05$ vs. control group pre-surgery; ${ }^{\#} \mathrm{p}<0.05$ vs. observation group pre-surgery). NF- $\mathrm{kB}$, nuclear factor- $\mathrm{\kappa B}$.

using independent-samples t-test. Serum levels of NF- $\kappa$ B, MMP-9 and MCP-1 at different time-points were compared using repeated measure analysis of variance (ANOVA). Count data are expressed as cases or $\%$, and comparison between groups was performed using $\chi^{2}$ test. Ranking data were compared using the rank-sum test. $\mathrm{P}<0.05$ was considered to indicate a statistically significant difference.

\section{Results}

Surgery success rate and complication incidence. There was no significant difference in the success rate between the two groups $(p>0.05)$. The incidence of complications in the observation group was lower than that in the control group ( $\mathrm{p}<0.05$; Table II).

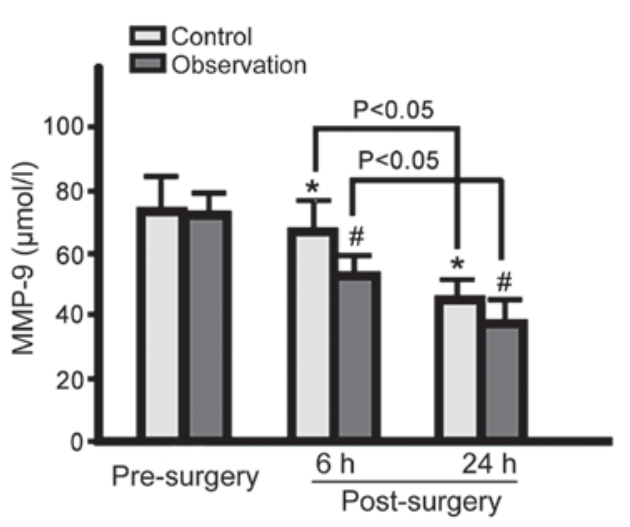

Figure 2. Serum MMP-9 levels in the control and observation groups pre- and post-surgery. Serum MMP-9 levels in both groups gradually decreased at 6 and 24 h post-surgery, with serum MMP-9 levels significantly lower in the observation group ( ${ }^{*} \mathrm{p}<0.05$ vs. control group pre-surgery; ${ }^{\#} \mathrm{p}<0.05$ vs. observation group pre-surgery). MMP-9, matrix metalloproteinase-9.

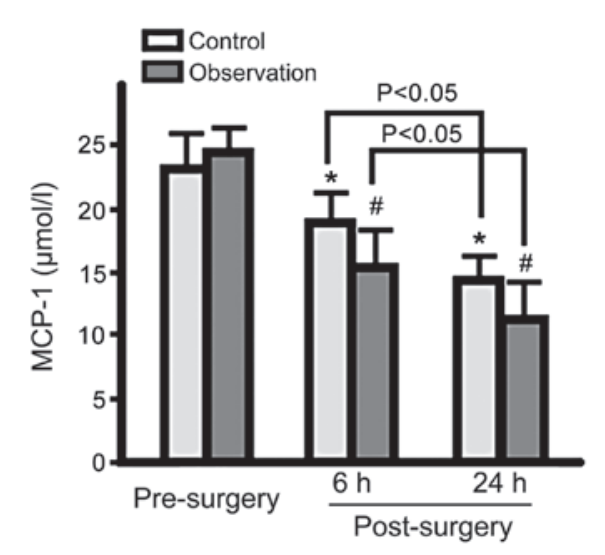

Figure 3. Serum MCP-1 levels in the control and observation groups pre- and post-surgery. Serum MCP-1 levels in both groups gradually decreased at 6 and 24 h post-surgery, with serum MCP-1 levels significantly lower in the observation group ( $\mathrm{p}<0.05$ vs. control group pre-surgery; ${ }^{\#} \mathrm{p}<0.05$ vs. observation group pre-surgery). MCP-1, monocyte chemoattractant protein-1.

GOS score. The GOS score of the observation group was significantly improved compared to the control group $(\mathrm{p}<0.05$; Table III).

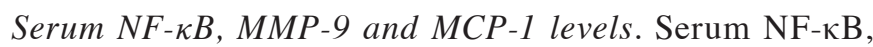
MMP-9 and MCP-1 levels in both groups were decreased at 6 and 24 h post-surgery. Observation group levels were lower for the 3 proteins than control group levels ( $p<0.05$; Figs. $1-3$ ). 


\section{Discussion}

Studies have shown that the peak period of rebleeding is 4-9 days post-cerebral aneurysm rupture with high mortality rate $(8,9)$. Early surgery is recommended for patients with Hunt-Hess grading levels I and II, but surgical timing for the patients with levels III to V remains controversial. Some scholars believe that early surgery may increase the mortality rate due to unstable condition induction (9). However, with the development of vascular intervention techniques and micro-technology, early (within $72 \mathrm{~h}$ ) and ultra-early (24 h) surgical treatment has become possible (10). Without the need for craniotomy, interventional embolization only causes small trauma and can be applied to patients with unstable condition. Interventional embolization leads to relatively fewer hemodynamic changes and stress responses, which in turn increases the success rate (11). The main drawback of interventional embolization is the need to accurately assess the distribution of intracranial blood vessels and aneurysms, and surgical failure is mainly due to vessel variation, errors in localizing target vessels, and incomplete embolization (12). The main complications are angiorrhea and vasospasm, which are related to mechanical stimulation and foreign body reactions caused by surgical materials (13).

After interventional embolization, the wound should be closed to prevent rebleeding, with hematoma removal, decompressive craniectomy, or extraventricular drainage then performed to stabilize the patient condition (13). After microsurgical neck clipping, aneurysms shrink due to fibrosis and the incidence of rebleeding is low. The use of microscopy provides clear visualization, which is helpful for avoiding the intracranial nerve and vascular injury, reducing bleeding and increasing positioning accuracy (14). In this study, no significant difference was found in surgical success rate between the two groups, while lower complication incidence rates and higher GOS scores were found in the observation group, indicating that early microsurgical neck clipping was safe and effective for the treatment of cerebral aneurysm rupture. In addition, studies have shown that depressurization treatment should not be performed during surgery to reduce the occurrence of postoperative serious complications, such as cerebral infarction (15). Important traffic arteries should not be clipped during surgery to reduce the occurrence of permanent dysfunction (16).

Our study showed that serum levels of NF- $\mathrm{kB}$, MMP-9 and MCP-1 in both groups gradually decreased at 6 and $24 \mathrm{~h}$ after surgery, with the observation group presenting significantly lower levels of all three proteins. Aneurysm formation is caused by the local expansion of the arterial wall and damage to elastic and collagen fibers (17). MMPs are mainly responsible for the degradation of arterial wall elastin and collagen (17), and inflammatory cells are the main source of MMP-9 in the arterial wall. The aggregation of macrophages in the aneurysm wall is the main intermediate step of the inflammatory response process (18). MCP-1 is a member of the chemokine CC subfamily and is the strongest monocyte-macrophage chemokine (19). Acute aneurysms are often accompanied with increased MCP-1 levels, which is consistent with the degree of inflammatory response. NF- $\mathrm{\kappa B}$ is widely involved in the inflammatory response, immune response, cell differentiation, apoptosis and stress responses. NF- $\mathrm{KB}$ can bind to the promoter regions of a variety of proinflammatory cytokines and immunomodulatory factors and is key player in the regulation of the expression of proinflammatory genes. After activation, NF- $\mathrm{KB}$ is transported into the nucleus to activate the expression of downstream target genes, which in turn induces macrophage aggregation in the aneurysm wall and the degradation of the extracellular matrix $(20,21)$. The changes in indicator protein expression observed in this study demonstrated that microsurgical treatment can significantly reduce the degree of the inflammatory response and reduce the expression level of MMP-9.

In conclusion, the application of early microsurgical neck clipping in the treatment of cerebral aneurysm rupture can reduce the incidence of complications and improve clinical prognosis, which may be associated with a decreased serum inflammatory response and reduced expression level of MMP-9. The shortcomings of the study were the small sample size and short follow-up time. In addition, the correlations between the expression levels of NF-кB, MMP-9 and MCP-1 were not analyzed. Further validation is needed.

\section{References}

1. Liu H, Zhang T, Jiao S, Li B, Guan J and Wang YX: Epidemiological investigation of 264 sporadic cases of ruptured cerebral aneurysm at a single institution in southwest China. Neuropsychiatr Dis Treat 11: 1609-1614, 2015.

2. Chalouhi N, Ali MS, Starke RM, Jabbour PM, Tjoumakaris SI, Gonzalez LF, Rosenwasser RH, Koch WJ and Dumont AS: Cigarette smoke and inflammation: Role in cerebral aneurysm formation and rupture. Mediators Inflamm 2012: 271582, 2012.

3. Liu Z, Wang Q, Ren J, Assa CR, Morgan S, Giles J, Han Q and Liu B: Murine abdominal aortic aneurysm model by orthotopic allograft transplantation of elastase-treated abdominal aorta. J Vasc Surg 62: 1607-1614, 2015.

4. Duellman T, Warren CL, Peissig P, Wynn M and Yang J: Matrix metalloproteinase-9 genotype as a potential genetic marker for abdominal aortic aneurysm. Circ Cardiovasc Genet 5: 529-537, 2012.

5. Yan YW, Fan J, Bai SL, Hou WJ, Li X and Tong H: Zinc prevents abdominal aortic aneurysm formation by induction of A20-mediated suppression of NF- $\mathrm{B}$ pathway. PLoS One 11: e0148536, 2016.

6. Grasso G and Perra G: Surgical management of ruptured small cerebral aneurysm: Outcome and surgical notes. Surg Neurol Int 6: 185, 2015.

7. Kim YB, Hong CK, Chung J, Joo JY and Huh SK: Long-term clinical and angiographic outcomes of wrap-clipping strategies for unclippable cerebral aneurysms. Yonsei Med J 55: 401-409, 2014.

8. Yamada Y, Kato Y, Ishihara K, Ito K, Kaito T, Nouri M, Oheda M, Inamasu J and Hirose Y: Role of endoscopy in multimodality monitoring during aneurysm surgery: A single center experience with 175 consecutive unruptured aneurysms. Asian J Neurosurg 10: 52, 2015.

9. Carter BS, Farrell C and Owen C: Microsurgical clip obliteration of middle cerebral aneurysm using intraoperative flow assessment. J Vis Exp 31: 1294, 2009.

10. Matsumura N, Shibata T, Hori E, Kamiyama H, Tani M, Okamoto S, Kubo M, Horie Y, Endo S and Kuroda S: An operating microscope with higher magnification and higher resolution for cerebral aneurysm surgery: Preliminary experience-technical note. Neurol Med Chir (Tokyo) 54: 497-501, 2014.

11. Cheikh A, Rachid R, Jehanne A, Adil A, Ali B, Cherrah Y, Amine EH and Abdeljalil EQ: Cost of treatment of cerebral aneurysm embolization: Study of associated factors. Neurol Ther 5: 145-154, 2016

12. Sato E, Konishi Y, Shimada A, Komatsubara K, Yazaki H, Fujitsuka $M$ and Shiokawa Y: Applications and roles of coil embolization and/or clipping in the treatment of cerebral aneurysm. Interv Neuroradiol 12 (Suppl 1): 77-85, 2006. 
13. Chung SW, Baik SK, Kim Y and Park J: Thromboembolic events after coil embolization of cerebral aneurysms: Prospective study with diffusion-weighted magnetic resonance imaging follow-up. J Korean Neurosurg Soc 43: 275-280, 2008.

14. Alaraj A, Luciano CJ, Bailey DP, Elsenousi A, Roitberg BZ, Bernardo A, Banerjee PP and Charbel FT: Virtual reality cerebral aneurysm clipping simulation with real-time haptic feedback. Neurosurgery 11 (Suppl 2): 52-58, 2015.

15. Jang EW, Kim YB, Chung J, Suh SH, Hong CK and Joo JY: Clinical risk factors affecting procedure-related major neurological complications in unruptured intracranial aneurysms. Yonsei Med J 56: 987-992, 2015.

16. Ha SK, Lim DJ, Seok BG, Kim SH, Park JY and Chung YG: Risk of stroke with temporary arterial occlusion in patients undergoing craniotomy for cerebral aneurysm. J Korean Neurosurg Soc 46: 31-37, 2009.

17. Ho WM, Chen CM, Lee YS, Chang KH, Chen HW, Chen ST and Chen YC: Association of MMP-9 haplotypes and TIMP-1 polymorphism with spontaneous deep intracerebral hemorrhage in the Taiwan population. PLoS One 10: e0125397, 2015.
18. Nowicki KW, Hosaka K, He Y, McFetridge PS, Scott EW and Hoh BL: Novel high-throughput in vitro model for identifying hemodynamic-induced inflammatory mediators of cerebral aneurysm formation. Hypertension 64: 1306-1313, 2014.

19. Hudson JS, Hoyne DS and Hasan DM: Inflammation and human cerebral aneurysms: Current and future treatment prospects. Future Neurol 8: 10, 2013.

20. Chalouhi N, Ali MS, Jabbour PM, Tjoumakaris SI, Gonzalez LF, Rosenwasser RH, Koch WJ and Dumont AS: Biology of intracranial aneurysms: Role of inflammation. J Cereb Blood Flow Metab 32: 1659-1676, 2012

21. Fennell VS, Kalani MY, Atwal G, Martirosyan NL and Spetzler RF: Biology of saccular cerebral aneurysms: A review of current understanding and future directions. Front Surg 3: $43,2016$. 